\title{
A rare combination of type 3 autoimmune polyendocrine syndrome (APS-3) or multiple autoimmune syndrome (MAS-3)
}

\author{
Corrado Betterle $\cdot$ Silvia Garelli • Graziella Coco • \\ Patrizia Burra
}

Received: 12 September 2013/ Accepted: 18 November 2013/Published online: 11 February 2014

(C) Springer-Verlag Italia 2014

\begin{abstract}
Context Type 3 autoimmune polyendocrine syndrome (APS-3) is defined by the presence of an autoimmune thyroid disease and another autoimmune illness, excluding Addison's disease; this is a frequent combination.

Case presentation We report the case of a 55 years old female patient with APS-3, with seven clinical or latent autoimmune manifestations. At 49 years of age she was admitted at the General Hospital for leukopenia, weight loss, tremors, anxiety and diarrhea. The personal history revealed ulcerative colitis and, during the last year, episodes of fever with migrant arthralgia and cutaneous lesions. The patient was evaluated for thyroid function and imaging, mielobiopsy, glycaemic control, gastrointestinal and rheumatologic disorders with specific biochemical tests, imaging and endoscopic procedures. We concluded that the patient was affected by APS-3, characterized by the association of Graves' disease, autoimmune leukopenia, latent autoimmune diabetes of the adult (LADA), autoimmune gastritis, ulcerative colitis, Sjögren's and antiphospholipid syndromes. The patient started low doses of corticosteroid drugs for leukopenia, underwent ${ }^{131}$ I therapy for hyperthyroidism and later started substitutive thyroid therapy with L-thyroxine, insulin therapy for LADA,
\end{abstract}

G. Coco prematurely dead on 2009 at 31 years of age.

C. Betterle $(\bowtie) \cdot$ S. Garelli $\cdot$ G. Coco

Endocrine Unit, Department of Medicine, University of Padova, Via Ospedale Civile 105, 35100 Padua, Italy

e-mail: corrado.betterle@unipd.it

P. Burra

Gastroenterology Unit, Department of Surgery, Oncology and Gastroenterology, University of Padova, Via Giustiniani 2, 35100 Padua, Italy mesalazine for ulcerative colitis and artificial tears for Sjögren's syndrome.

Conclusions In this article we report a complex case of APS-3, characterized by the association of seven different autoimmune diseases, which required a complex therapeutic strategy.

Keywords Autoimmune polyendocrine syndrome type 3 - Multiple autoimmune syndrome type 3 . Graves' disease - Diabetes mellitus type 1 . Autoimmune gastrointestinal disorders

\section{Introduction}

In 1980 Neufeld and Blizzard [1] proposed the classification of the so called autoimmune polyendocrine syndromes (APS) based on clinical criteria and four main types of APS (type 1 , type 2 , type 3 , type 4 ) were identified (Table 1 ).

As regard APS type 3 (APS-3), the same authors subdivided it in three subgroups, based on the association of one thyroid autoimmune disease (TAD) (Hashimoto's thyroiditis, Graves' disease, endocrine ophthalmopathy or pretibial myxoedema) with one or more of the following diseases: diabetes mellitus type 1 (DM-1) (3A), chronic atrophic gastritis or pernicious anemia (3B), and vitiligo, alopecia or myasthenia gravis (3C).

In the course of the subsequent 40 years the number of autoimmune diseases has greatly increased, and on the book published on the occasion of the 50th anniversary from the discovery of autoimmune diseases, about 80 diseases were included [2]. For these reasons we revised and expanded the classification of APS-3 into four subgroups, practically including all autoimmune diseases 
Table 1 Classification of autoimmune polyendocrine syndromes (APS) modified from Neufeld [1]

\begin{tabular}{|c|c|}
\hline $\begin{array}{l}\text { APS-1 or APECED } \\
\text { (autoimmune-poly-endocrine- } \\
\text { candidiasis-ectodermal- } \\
\text { dystrophy) }\end{array}$ & $\begin{array}{l}\text { Chronic mucocutaneous } \\
\text { candidiasis } \\
\text { Chronic hypoparathyroidism } \\
\text { Addison's disease (at least two out } \\
\text { of three) }\end{array}$ \\
\hline APS-2 or Schmidt's syndrome & $\begin{array}{l}\text { Addison's diseases }+ \text { thyroid } \\
\text { autoimmune disease and/or } \\
\text { diabetes mellitus type } 1\end{array}$ \\
\hline $\begin{array}{l}\text { APS-3 (excluding Addison's } \\
\text { disease) }\end{array}$ & $\begin{array}{l}\text { Thyroid autoimmune disease }+ \\
\text { DM-1: Type 3A } \\
\text { Chronic atrophic gastritis or } \\
\text { pernicious anemia: Type 3B } \\
\text { Vitiligo, alopecia or myasthenia } \\
\text { gravis: Type 3C }\end{array}$ \\
\hline APS-4 & $\begin{array}{l}\text { Any other possible association of } \\
\text { autoimmune diseases }\end{array}$ \\
\hline
\end{tabular}

associated to TADs (with the exception of AD) (Table 2) [3].

Subsequently some authors proposed a simpler classification in APS type 1 and type 2, because of similar genetic background and pathophysiology between APS type 2, type 3 and type 4. In 2004 Eisenbarth et al. [4] defined the two parties as "splitters" and "lampers", being in favour of a simpler classification. In our opinion, the classification proposed by Neufeld and Blizzard and completed by us [1, $3]$ is more practical from a clinical and methodological point of view.
Furthermore, the term APS is not fully appropriate, because it is often used to indicate not only cases of multiple autoimmune endocrine diseases (such as TADs and DM-1), but also associations between endocrine and nonendocrine autoimmune diseases (such as DM-1 with celiac disease or TADs and gastric autoimmunity), or the associations between non-endocrine autoimmune diseases (for instance vitiligo and myasthenia gravis, or autoimmune hepatitis and Sjögren's syndrome). For these reasons, we recently proposed to substitute or to associate to the term APS the term Multiple Autoimmune Syndromes (MAS), because we consider the last a more appropriate term to denote these autoimmune overlapping conditions $[5,6]$.

In this article we present a complex case of a patient affected by APS/MAS-3 with Graves' disease and six other different clinical or latent autoimmune diseases.

\section{Case presentation}

Our patient is a 55 years old Caucasian woman. The family history revealed chronic thyroiditis with hypothyroidism in the mother and in a sister; an aunt and a cousin were affected by Graves' disease, another relative had diabetes mellitus type 2 and a grandfather had Bürger's disease.

Anamnestically, she referred tonsillectomy at the age of 10 years. At 36 years diagnosis of ulcerative colitis was performed. From 36 years she took mesalazine therapy, which was finally stopped for prolonged remission at 46 years of age.

Table 2 The combinations of different autoimmune diseases present in our patient (in italics), with other possible diseases present in a patient with APS/MAS-3

\begin{tabular}{|c|c|c|c|}
\hline Type 1 DM (LADA) & $\begin{array}{l}\text { Chronic atrophic gastritis } \\
\text { Ulcerative colitis }\end{array}$ & Autoimmune leukopenia & $\begin{array}{l}\text { Sjögren's syndrome } \\
\text { Anti-phospholipid syndrome }\end{array}$ \\
\hline Hirata's syndrome & Pernicious anemia & Vitiligo & Systemic lupus erythematodes \\
\hline Premature ovarian failure & Celiac disease & Alopecia & Discoid lupus erythematodes \\
\hline Adeno-hypophysitis & Other inflammatory bowel diseases & Bullous Skin diseases & Rheumatoid arthritis \\
\hline Neuro-hypophysitis & Primary biliary cirrhosis & Chronic idiopathic urticaria & Mixed connective tissue disease \\
\hline Chronic hypoparathyroidism & $\begin{array}{l}\text { Autoimmune hepatitis } \\
\text { Sclerosing cholangitis } \\
\text { Autoimmune pancreatitis }\end{array}$ & $\begin{array}{l}\text { Myasthenia gravis } \\
\text { Stiff-person syndrome } \\
\text { Multiple sclerosis } \\
\text { Autoimmune anemia } \\
\text { Autoimmune thrombocytopenia }\end{array}$ & $\begin{array}{l}\text { Seronegative arthritis } \\
\text { Systemic sclerosis } \\
\text { Dermato/polymyositis } \\
\text { Vasculitis }\end{array}$ \\
\hline Endocrine glands & Gastrointestinal tract and liver & $\begin{array}{l}\text { Skin } \\
\text { Muscles } \\
\text { Nervous system } \\
\text { Haematologic system }\end{array}$ & $\begin{array}{l}\text { Autoimmune rheumatic disorders } \\
\text { Vasculitis }\end{array}$ \\
\hline $3 \mathrm{~A}$ & $3 \mathrm{~B}$ & $3 \mathrm{C}$ & $3 \mathrm{D}$ \\
\hline
\end{tabular}

APS/MAS-3 autoimmune polyendocrine syndrome/multiple autoimmune syndrome type 3 
At 48 years she referred episodes of fever up to $39^{\circ} \mathrm{C}$, migrant arthralgia and cutaneous vasculitic lesions; at that time the patient was taking no drug. Blood tests revealed severe leukopenia $\left(870 \mathrm{WBC} / \mathrm{mm}^{3}\right)$ and anti-nuclear antibodies (ANA) positivity (titer of 1:320 with an homogeneous pattern), whereas extractable nuclear antigen antibodies (ENA) were negative. So, a therapy based on antibiotics and granulocyte stimulating growth factor was started, with good acute clinical response; subsequently, leukopenia was stabilized $\left(2,500 \mathrm{WBC} / \mathrm{mm}^{3}\right)$ with low doses of prednisone. A bone marrow needle aspiration was performed, revealing myeloid hyperplasia with reduced number of mature cells: diagnosis of autoimmune leukopenia was established on the basis of chronic presence of ANA positivity, response to steroid therapy and the exclusion of other possible causes.

At 49 years the patient was admitted to the Gastroenterology Division of Padova General Hospital for weight loss and diarrhea. The clinical examination showed the presence of anxiety, tremors and goiter without ophtalmopathy. Hormonal values showed TSH inhibition with high free T4 and free T3 levels, and positivity to antibodies against thyroperoxidase (TPOAbs), thyroglobulin (TgAbs) and TSH receptor (TRAb). Thyroid Doppler-US scan revealed increased gland volume, inhomogeneous hypoechoic structure and intense hypervascularization; an ${ }^{\mathrm{m} 99} \mathrm{Tc}$ scintigraphy showed increased uptake, so the diagnosis of Graves' disease was established. The patient had persistent modest leukopenia under low doses of prednisone.

Because of leukopenia anti-thyroid drugs weren't started, and the patient was initially treated with symptomatic drugs, such as beta-blockers, and after few weeks with ${ }^{131} \mathrm{I}$ therapy. After few more weeks she developed persistent hypothyroidism and substitutive therapy with L-thyroxine (75 gamma/die) was started, with normalization of the thyroid function.

Furthermore, on this occasion microcytic anemia with hyposideremia and thrombocytopenia $\left(121,000 / \mathrm{mm}^{3}\right)$ were also demonstrated in addition to leukopenia. In the suspect of an autoimmune gastritis, specific blood tests revealed the presence of high titers of gastric parietal cells antibodies (PCA), high levels of both gastrinemia and chromogranine A, with negativity for intrinsic factor autoantibodies and tissue transglutaminase of IgA class.

An esophagogastroduodenoscopy (EGDS) was performed and revealed macroscopic evidence of atrophic body gastritis; histological examination confirmed the presence of body and fundus atrophy with intestinal metaplasia, hyperplasia of the enterochromaffin cells without Helicobacter pylori infection, while the duodenal biopsy was negative. These data confirmed the presence of autoimmune gastritis. Oral iron replacement therapy was started.
To further investigate thrombocytopenia, the patient underwent an ultrasound examination of the abdomen, which showed an enlarged spleen (diameter $16 \mathrm{~cm}$ ) with an homogenous pattern. On this occasion, low titers of anticardiolipin antibodies (IgM and IgG classes) and antibeta2-glycoprotein I (IgM class) were documented. High titers $(1 / 640)$ of ANA were confirmed with homogeneous pattern, but double strain DNA antibodies (dsDNA Abs) and anti-platelet antibodies were negative.

On this occasion hyperglycemia $(6.94 \mathrm{mmol} / \mathrm{L})$ was discovered for the first time, with high levels of HbA1c $(59 \mathrm{mmol} / \mathrm{mol}, 7.5 \%)$. For this reason autoantibodies to endocrine pancreas were investigated: high titers $(1 / 160$ U/JDF) of islet-cells antibodies (ICA) and glutamic acid decarboxylase autoantibodies (GADAbs) (12.1 U/mL) were identified, while autoantibodies to second islet antigen (IA2Abs) were negative. On the basis of these data, the diagnosis of latent autoimmune diabetes of the adult (LADA) was performed and low dosage insulin therapy was started. During the subsequent years, the patient reported neurological disturbances, simulating sciatic nerve inflammation, and she was treated with anti-inflammatory drugs with no beneficial effect. Later on, the diagnosis of diabetic neuropathy was performed and alpha-lipoic-acid was started with good symptomatic control after few weeks of therapy.

The patient referred also mouth and eye dryness. A Schirmer's test revealed a diminished tear secretion. Autoantibodies to ENA were confirmed to be negative, but a salivary gland US-scan revealed a typical Sjögren's syndrome pattern. No biopsies of minor salivary glands were performed. A topic treatment with artificial tears was started.

During this hospitalization in Gastroenterology Unit, a colonoscopy was also repeated, and revealed signs of silent pancolitis.

The HLA typing of the patient revealed this pattern: A24(9), 26(10); B44(12), 62(15); Cw7; (Bw4); DRB1* 04, 13; DQA1* 0303/0505; DQB1* 0302/0301.

The patient was followed-up by a team of specialists: endocrinologist, diabetologist, gastroenterologist, rheumatologist and haematologist. During the following years she felt quite good, taking previously mentioned medications.

At 55 years she manifested once again gastrointestinal symptoms with fecal blood loss and consequent severe anemia, therefore she underwent colonoscopy which revealed reactivation of ulcerative colitis, indicating the need to start again with mesalazine. After few weeks of therapy she achieved a complete remission of symptoms.

In the same occasion the patient repeated EGDS. Gastric histology showed mild metaplastic-atrophic gastritis, classified as stage II by OLGA System 2005; Helicobacter 
pylori was negative. So the histological features confirmed the autoimmune gastritis.

The patient underwent biochemical and hormone assessment, showing stable hemoglobin $(12 \mathrm{~g} / \mathrm{L})$, with good renal and hepatic function. Positivity of gastric mucosa antibodies was reported, as well as increase of gastrin. Low levels of 25-hydroxy vitamin D3 were detected, so an oral supplementation with cholecalciferol was started.

The patient is still on low dose of steroids, mesalazine, insulin, L-thyroxine.

\section{Discussion}

TADs are the most frequent autoimmune diseases in the world population, presenting in the majority of cases as spontaneous hypothyroidism, being present in 4-21\% of females and in 3-16\% of males depending on age; hyperthyroidism is rarer, affecting about $0.5 \%$ of population [7, 8]. Furthermore, autoantibodies directed to thyroid antigens have been described in $11 \%$ of euthyroid subjects [9]. About $15 \%$ of the patients with TADs is affected by another clinical autoimmune disease [10] and another $15 \%$ of those with apparently isolated TADs may result positive if screened for organ and non-organ-specific autoantibodies. So, we may conclude that about $30 \%$ of patients with TADs is affected by a clinical, subclinical or potential APS/ MAS-3. For this reason APS/MAS-3 is the most common APS/MAS, being present in about $5 \%$ of the female population and $1.5 \%$ of males [6].

The majority of APS/MAS-3 patients presents TADs associated with only another autoimmune disease, and the most common associations are with chronic atrophic gastritis (39\% of the cases) [11] or with DM-1 (20-30\% of the cases) $[12,13]$. To our knowledge, only four patients have been described so far with three or more autoimmune diseases complicating TADs. The first was described in Japan and was affected by Graves' disease with other five autoimmune diseases, i.e. DM-1, alopecia areata, autoimmune neutropenia, Sjögren's syndrome and chronic cutaneous lupus erythematosus [14]. Another patient coming from Greece presented Hashimoto's thyroiditis associated with other three autoimmune diseases: DM-1, atrophic gastritis with pernicious anemia and immunologic thrombocytopenic purpura [15]. The third patient was described by American colleagues with silent thyroiditis and other three autoimmune diseases, i.e. isolated corticotropin deficiency, ulcerative colitis and alopecia universalis [16]. An Italian patient with Down's syndrome was described having TAD (Hashimoto's thyroiditis followed by Graves' disease) with three other diseases, i.e. arthropathy, DM-1 and celiac disease [17].

Our patient had seven autoimmune manifestations and fulfill all four subgroups of APS/MAS-3, being affected by Graves' disease and LADA (3A), autoimmune gastritis and ulcerative colitis (3B), autoimmune neutropenia (3C), Sjögren's syndrome and anti-phospholipids antibodies with thrombocytopenia (3D) (Table 2). Six diseases were clinically evident and one (anti-phospholipid syndrome) was latent, not having clinical symptoms but only autoantibodies and thrombocytopenia.

The management of our patient needed the intervention and cooperation of many different specialists, from endocrinologist to diabetologist, gastroenterologist, rheumatologist and haematologist. Only a multidisciplinary setting allowed to minimize therapies, controlling different illnesses symptoms and reducing drugs side effects.

In patients with TADs without other clinical autoimmune manifestations, we suggest not to perform a universal autoantibodies screening, but to limit the number of exams depending on the family history of the single patient and on the most common associations (chronic atrophic gastritis, DM-1, Sjögren's syndrome and celiac disease); obviously, clinical suspect is fundamental. In the case of negativity, patients should be re-evaluated every 2 or 3 years. This approach permits to identify the eventual ongoing new autoimmune diseases and, if needed, to precociously treat the patient.

In conclusion, in this paper we described a complex case of APS/MAS-3 with seven different diseases, which required a complex therapeutic strategy taking into account adverse effects of drugs.

Conflict of interest Betterle C, Garelli S and Burra P declare that they have no conflict of interest. Coco $\mathrm{G}$ had no conflict of interest.

Animal rights As regards the patient described in the submitted case report "A rare combination of type 3 autoimmune polyendocrine syndrome (APS-3) or multiple autoimmune syndrome (MAS-3)", clinical procedures were in accordance with the ethical standards of the responsible committee on human experimentation (institutional and national) and with the Helsinki Declaration of 1975, as revised in 2000 and 2008.

Informed consent As regards the submitted case report "A rare combination of type 3 autoimmune polyendocrine syndrome (APS-3) or multiple autoimmune syndrome (MAS-3)", written consent has been obtained from the patient.

\section{References}

1. Neufeld M, Blizzard RM (1980) Polyglandular autoimmune diseases. In: Pinchera A, Doniach D, Fenzi GF, Baschieri L (eds) Symposium on autoimmune aspects of endocrine disorders. Academic Press, New York, pp 357-365 
2. Rose N, MacKay IR (eds) (2006) The autoimmune diseases, 4th edn. Academic Press, St. Louis

3. Betterle C, Dal Pra C, Mantero F, Zanchetta R (2002) Autoimmune adrenal insufficiency and autoimmune polyendocrine syndromes: autoantibodies, autoantigens, and their applicability in diagnosis and disease prediction. Endocr Rev 23:327-364

4. Eisenbarth GS, Gottlieb PA (2004) Autoimmune polyendocrine syndromes. N Engl J Med 350:2068-2079

5. Betterle C, Presotto F (2008) Autoimmune polyendocrine syndromes (APS) or multiple autoimmune syndromes (MAS). In: Walker S, Jara LJ (eds) Handbook of systemic autoimmune diseases, endocrine manifestations of systemic autoimmune diseases, vol 9. Elsevier, Amsterdam, pp 135-148

6. Betterle C, Presotto F (2009) Sindrome autoimmune multipla di tipo 3: una galassia in espansione. L'Endocrinologo 10:132-142

7. Canaris GJ, Manowitz NR, Mayor G, Ridgeway ED (2000) The Colorado thyroid disease prevalence study. Arch Intern Med 160:526-534

8. Weetman AP (2000) Graves' disease. N Engl J Med 343:1236-1248

9. Hollowell JG, Staehling NW, Flanders WD, Hannon WH, Gunter EW, Spencer CA, Braverman LE (2002) Serum TSH, T4 and thyroid antibodies in the United States population 1998 to 1994 National Health Nutrition Examination Survey (NHANES III). J Clin Endocrinol Metab 87:489-499

10. Boelaert K, Newby PR, Simmonds MJ, Holder RL, Carr-Smith JD, Heward JM, Manji N, Allahbadia A, Armitage M, Chatterjee KV, Lazarus JH, Pearce SH, Vaidya B, Gough SC, Franklyn JA (2010) Prevalence and relative risk of other autoimmune diseases in subjects with autoimmune thyroid disease. Am J Med 123:1-9
11. Lahner E, Centanni M, Agnello G, Gargano L, Vannella L, Iannoni C, Delle Fave G, Annibale B (2008) Occurrence and risk factors for autoimmune thyroid disease in patients with atrophic body gastritis. Am J Med 121:136-141

12. Van den Driessche A, Eenkhoorn V, Van Gaal L, De Block C (2009) Type I diabetes and autoimmune polyglandular syndrome: a clinical review. Neth J Med 67:376-387

13. Warncke K, Frohlich-Reiterer EE, Thon A, Hofer S, Wiemann D, Holl RW (2010) Polyendocrinopathy in children, adolescents and young adults with type 1 diabetes. Diabetes Care 33:2010-2012

14. Shimomura H, Nakase Y, Furuta H, Nishi M, Nakao T, Hanabusa T, Sasaki H, Okamoto K, Furukawa F, Nanjo K (2003) A rare case of autoimmune polyglandular syndrome type 3 . Diabetes Res Clin Pract 61:103-108

15. Elefsiniotis IS, Papatsimpas G, Liatsos GD, Tasidou A, Moulakakis A (2008) Atypical autoimmune polyglandular syndrome type 3 overlapped by chronic HCV infection resulting in carcinogenesis and fatal infection. South Med J 101:756-758

16. Sheehan MT, Islam R (2009) Silent thyroiditis isolated corticotropin deficiency and alopecia universalis in a patients with ulcerative colitis and levated levels of factor VIII: an unusual case of autoimmune polyglandular syndrome type 3 . Endocr Pract 15:138-142

17. Salzano G, Lombardo F, Arrigo T, Sferlazzas C, Wasniewska M, Valenzise M, De Luca F (2010) Association of five autoimmune diseases in a young woman with Down's syndrome. J Endocrinol Invest 33:202-203 